DOI: $10.25100 /$ pfilosofica.v0i50.8961

\title{
LA INSTITUCIÓN DEL ESTADO COMO UNA VELUTI MENTE
}

Iván Ramón Rodríguez Benavidez

Universidad de La Salle, Bogotá, Colombia.

\begin{abstract}
Resumen
La exposición de los regímenes políticos del Tratado Político no es aleatoria; Spinoza expone en primer lugar el que menos conviene, esto es, el monárquico, después el que conviene más, la aristocracia, y finalmente la democracia, régimen al que concibe como el más perfecto. Este criterio de exposición no sirve de criterio para investigar cuál es el valor de la democracia para Spinoza, pero nos permite investigar cuál es el principal obstáculo o peligro que debe enfrentar la democracia para que se pueda instituir realmente por parte de los ciudadanos, y para que no sea instrumentalizada por los intereses particulares de los gobernantes y políticos de turno.
\end{abstract}

Palabras clave: democracia; libertad; engaño; acuerdo; deliberación.

Cómo citar este artículo: Rodríguez Benavidez, I. R. (2020). La institución del estado como una veluti mente. Praxis Filosófica, (50S), 59-72. doi: 10.25100/pfilosofica.v0i50.8961.

Recibido: 5 de noviembre de 2019. Aprobado: 27 de diciembre de 2019 . 


\title{
The Institution \\ of the State as a veluti mente
}

\section{Iván Ramón Rodríguez Benavidez ${ }^{1}$}

\begin{abstract}
The exposure of the political regimes of the Political Treatise is not random; Spinoza first exposes the least convenient, that is, the monarchist, then the one that suits the most, the aristocracy, and finally democracy, a regime he conceives as the most perfect. This exposure criterion does not serve as a criterion to investigate what is the value of democracy for Spinoza, but it also allows us to investigate what is the main obstacle or danger that democracy must face so that it can really be instituted by citizens, and so that it is not instrumentalized by the particular interests of the rulers and politicians on duty.
\end{abstract}

Keywords: Democracy; Freedom; Deception; Agreement; Deliberation

${ }^{1}$ Doctor en filosofía por la Universidad de Barcelona. Magister en filosofía por la Pontifica Universidad Javeriana. Su área de estudio es la filosofía moderna. Profesor Asociado, Universidad de La Salle.

ORCID: 0000-0002-9182-7307 E-mail: ivrodriguez@unisalle.edu.co 


\title{
LA INSTITUCIÓN DEL ESTADO COMO UNA VELUTI MENTE
}

\author{
Iván Ramón Rodríguez Benavides
}

Universidad de La Salle, Bogotá, Colombia.

\section{Introducción}

Para el análisis de la concepción del Estado de Spinoza seguiremos las explicaciones que Spinoza presenta en el Tratado Politico (TP) y en el Tratado Teológico Político (TTP). No obstante, es pertinente tener en cuenta que entre los dos tratados se presenta una diferencia, puesto que mientras en el TTP Spinoza se refiere únicamente a la democracia, y parte de la certeza de que es el mejor régimen político de gobierno, en el $T P$ estudia las mejores condiciones de los tres regímenes conocidos; a saber, del monárquico, del aristocrático con sus variaciones para concluir que el mejor régimen es el democrático. De acuerdo con Vidal Peña los tres regímenes son tratados por Spinoza "con una especie de distanciamiento descriptivo (Spinoza habla de lo que ha de ser, en su género, cada una de esas formas de gobierno, para aproximarse más a la racionalidad)" (1974, p. 51).

La exposición de los regímenes políticos del Tratado Político no es aleatoria; Spinoza expone en primer lugar el que menos conviene, esto es, el monárquico, después el que conviene más, la aristocracia, y finalmente la democracia, régimen al que concibe como el más perfecto. Esto nos permite plantear varias preguntas: ¿Por qué en el TTP Spinoza asume inmediatamente la democracia como el mejor régimen? Esto lo preguntamos especialmente por la segunda pregunta que suscita la lectura del $T P$ ¿Por qué en el $T P$ Spinoza se ocupa de otros regímenes si ya sabe que la democracia es el 
mejor? ¿Cuál es el objetivo y el método de Spinoza en el $T P$, dado que las dos primeras preguntas supondrían que en el $T P$ no sigue un método deductivo sino más bien hermenéutico demostrativo?

Antes de iniciar con el análisis de las obras, y si tenemos en cuenta los comentarios que Spinoza expresa en sus tratados encontramos que manifiesta un optimismo de época en el TTP. Se siente afortunado de vivir en Holanda y en particular en Ámsterdam, puesto que es el país que se ha caracterizado por defender la libertad. Explícitamente en el prólogo del TTP, destaca la suerte que tiene de vivir en una época y en un lugar donde la libertad es defendida como el bien más valioso. Como ya se explicó, para cuando está escribiendo el TTP Spinoza participa de la vida política de Ámsterdam hasta el punto de comprometerse con el proyecto republicano de los hermanos de Witt. Con ellos defiende y promueve las ideas republicanas, que defienden y promueven la libertad, y se opone a la monarquía de los Orange porque considera que "en coalición con el clero calvinista, conspiraban para mantener al pueblo en un estado de ignorancia y superstición" (Smith, 2007, p. 172).

En cambio, en el TP Spinoza no muestra el mismo optimismo, quizás porque para entonces sus amigos, los hermanos de Witt, ya habían sido asesinados por el clan Orange. Del asesinato de los hermanos De Witt Spinoza obtiene dos grandes aprendizajes: en primer lugar que "había sobrestimado los efectos del argumento racional en las opiniones de las masa" (Balibar, 2011, p.133), y en segundo lugar que "había sobrestimado la capacidad de los hombres de establecer más allá de las circunstancias y en conformidad con la "naturaleza" un régimen democrático" (Balibar, 2011, p.133). Esta interpretación se entrevé en las críticas de Spinoza al gobierno de Jan Witt puesto que no tomó las precauciones necesarias para consolidar su modelo republicano. De otra parte, esta posición crítica permite comprender que Spinoza no "se identifica con la ideología y los intereses de los regentes" es decir con la familia Orange (Balibar, 2011, p.172).

\section{Estado}

En el TP Spinoza conserva la idea política del Estado, pero con un criterio más realista que permita tener presentes los peligros que enfrenta quien quiere fundar un Estado. Sin embrago, no podríamos decir que en el $T P$ Spinoza olvida por completo el optimismo. Este cambio se observa en tanto que en el TP Spinoza problematiza aún más sus reflexiones políticas como lo veremos más adelante, y estudia los otros regímenes para analizar sus posibles ventajas, pero ante todo para mostrar las desventajas de los regímenes monárquico y aristocrático frente al democrático. 
La asociación de individuos que pactaron, y que por consiguiente cedieron su poder, ha instituido el Estado. Spinoza sostiene tanto en la Ética, especialmente en la parte V, como en el TTP, que en el Estado el poder instituido se caracteriza por ser de forma colegiada; a este régimen en el que todos comparten el poder lo denomina democracia, además, como se dijo antes es el único régimen que estudia en el TTP. Spinoza define la democracia como "la asociación general de los hombres, que posee colegialmente el supremo derecho a todo lo que puede" (TTP, XVI, 193/20), asociación que, como se explicó antes, no es contradicción con el estado natural sino que emerge de los individuos para consolidar un poder colectivo (Gatens y Lloyd,1999, p.167). En el TP Spinoza parte de la certeza de que la democracia es el mejor régimen, puesto que es el régimen donde se legisla y se gobierna de forma colectiva, y de esta manera se logra que "insofar as they promote the freedom of each, they increase the power of all and this power of all is coextensive with the power of the polity" (Gatens y Lloyd, 1999, p.119).

Spinoza continúa su reflexión en torno a la idea del poder colegiado del Estado en el TP. En su segundo tratado parte de que todos los ciudadanos dependen jurídicamente de la sociedad, pero agrega respecto a lo planteado en el TTP que para alcanzar la realización del Estado cada ciudadano junto a los otros ciudadanos ha de comportarse "como una sola mente" (TP, III, 286/8; VIII, 326/20). Este punto de partida, que concibe la asociación política como "una sola mente" más que como un cuerpo político es uno de los ejes de mayor novedad en la reflexión política de Spinoza. La concepción del Estado como una sola mente nos permitirá posteriormente mostrar que en Spinoza se devela un pensamiento republicano más que un pensamiento liberal. A partir de este eje podemos plantear inmediatamente la pregunta siguiente: ¿por qué Spinoza concibe la asociación que resulta del pacto como una sola mente en lugar de concebirla solo como un cuerpo, que es la forma como solía concebirse el Estado en el pensamiento político de su época?; también nos preguntamos ¿cuál es la diferencia entre concebir el Estado como mente y como cuerpo?

Para dar curso a estas preguntas partimos de que Spinoza considera que "el poder humano debe ser valorado no tanto por la robustez del cuerpo como por la fortaleza del alma" (TP, II, 280/18), y que este principio también lo aplica al Estado. De esta manera se destaca que la fuerza del pacto no solo reside en el poder físico sino también en la capacidad de obrar conforme a la razón. Así, Spinoza no desconoce que el Estado necesita el poder (la fuerza física) para gobernar, pero advierte que el mero poder físico es susceptible de ser burlado. Por esta razón también considera que la institución del 
Estado no solo requiere de poder físico, también requiere de conocimiento puesto que, como lo explica en la Ética, los hombres actúan solo en tanto que entienden, y que actuar por virtud supone actuar guiado por la razón, ya que actuar conforme a la razón implica saber qué es lo que más conviene a la conservación y aumento del ser (E4P24). De la misma manera, el Estado tiene como fin contribuir a que los hombres cumplan con su fin, a saber, aumentar su conatus. En este sentido, Balibar sostiene que la razón spinoziana busca lo que es útil para sí misma y hace lo que sea necesario en orden a preservar su propia existencia (Balibar, 2009, p.55), y por lo tanto, la concepción del Estado como una sola mente busca reponder a la exigencia del conatus. De acuerdo con Balibar de esta forma se concibe la "razón como útil pero no instrumental" (Balibar, 2009, p.55), con lo cual Balibar nos aporta otro argumento para la comprensión de Spinoza; aunque los hombres se unen en el Estado con un solo fin, esto es aumentar su conatus, ese fin muestra que se puede hacer un uso utilitarista de la razón sin caer en un uso instrumental. Además, el fin de aumentar el conatus como razón de los individuos para asociarse nos permite comprender que la institución de la sociedad va más allá del temor a la muerte violenta, puesto que también se pacta, como lo citamos antes "para incrementar nuestra virtud y nuestra racionalidad" (Smith, 2007, p.184). Este es el conocimiento al que alude Spinoza cuando define el Estado como la acción de los ciudadanos que se comportan como una sola mente.

En el caso de un individuo particular el conocimiento hace que su actuar pueda comprenderse como un comportamiento en el que es causa de sus propias acciones; de hecho, en la Ética, Spinoza define la libertad precisamente como una acción en la que el hombre es causa adecuada de sus acciones. Ahora bien, si aceptamos que Spinoza aplica el mismo principio al Estado entonces para que el Estado sea libre es necesario que los ciudadanos actúen políticamente como una sola mente. Esto significa, por ahora, que todos persiguen el mismo fin con lo cual adquieren un poder colectivo, $\mathrm{y}$ que, además, el Estado sea causa adecuada de sus propias acciones. En otras palabras, el objeto de la institución del Estado para Spinoza consiste en hacer de los individuos aislados una sola sociedad y alcanzar por esta vía una fortitudo animi.

Cuando se comprende el valor de la asociación de los individuos en el Estado también se puede entender por qué para Spinoza lo opuesto al Estado es la soledad, el desierto. Spinoza insiste tanto en la Ética como en los dos tratados que "el miedo a la soledad es innato a todos los hombres" (TTP, VI, 297/19) y (E4P35S). Porque en soledad los hombres ni siquiera podrían cumplir la ley natural de conservar la propia existencia, puesto que 
ni tienen lo necesario para la vida ni pueden defenderse. Y como explica en (TP, IV, 293), incluso, un Estado que no logra que los súbditos vivan en paz y obedezcan la ley está más próximo al estado natural donde los hombres viven en soledad, que a un verdadero Estado. Ahora bien, si consideramos el principio de actuar de acuerdo con lo que es más útil, entonces, Spinoza explica que nada es más útil para los hombres que los mismos hombres (EIV18ESIII). Por esta razón en soledad el hombre no alcanzaría su utilidad, es decir, cumplir la exigencia del conatus. Esto conlleva a que, tal como lo propone Gatens, los hombres aislados no sean capaces de existir (Gatens y Lloyd, 1999, p.110); por lo tanto, lo opuesto a vivir en el Estado es vivir en la soledad lo que conduce a la imposibilidad de existir. Por todo esto en Spinoza no se puede hablar de una elección de vida fuera de la comunidad, puesto que "Human life is necessarily collective life" (Gatens y Lloyd, 1999, p. 110).

No obstante, la pregunta persiste: ¿cuál es la ganancia política del pensamiento colectivo frente al pensamiento aislado? Para responder esta pregunta aún necesitamos otras discusiones en torno a otros ejes del pensamiento político, pero adelantamos la respuesta: es la deliberación. Más adelante cuando expliquemos el valor de la deliberación comprenderemos completamente el sentido de unidad de mente que propone Spinoza para el Estado, pero antes de continuar en esa dirección es pertinente ocuparnos de una aparente contradicción.

\section{Contradicción entre libertad y obediencia}

El Estado, aunque se comprende como el poder compartido por todos, demanda de un soberano que lo represente, esto es, de la potestad suprema. Lo cual implica, por parte de los ciudadanos la obediencia que le deben a la potestad suprema instituida, pero este sometimiento a una potestad soberana parecería contradecir lo que se dijo antes, en cuanto los individuos no ceden todo su poder y lo que ceden no lo ceden de forma definitiva. En cambio ahora se exige que los hombres que cedieron su poder de defenderse no estén dispuestos a ejercerlo y acepten la mediación del Estado puesto que "cada ciudadano no es autónomo sino que depende jurídicamente de la sociedad, cuyos preceptos tiene que cumplir en su totalidad" (TP III, 286/8).

Tanto en el TTP como en el TP Spinoza concibe al individuo como un sujeto racional que sopesa sus decisiones en función de una utilidad, para obtener un bien mayor o para evitar el mal mayor y obtener el mal menor. El resultado de tal procedimiento le permite entender por qué obedecer la ley le es más conveniente aún si es una ley absurda (TP, III, 287; TTP, XVI 193,194 y 198; E4P73), por lo tanto aún en el caso en el que los ciudadanos 
obedecen leyes que se oponen a la razón, si la decisión es el resultado de una acto del entendimiento que permite comprender la utilidad de obedecer dicha ley en lugar de no cumplirla, entonces, en ese caso, obedecer no se opone a actuar de acuerdo con la propia razón y en consecuencia, tampoco implica una pérdida de libertad. Desde otra perspectiva puede concluirse que el solo hecho de encontrar una ley absurda no es argumento suficiente para no cumplirla o para acusar perdida de libertad, pues "ese perjuicio quedaría ampliamente compensado por el bien que surge del mismo estado político" (TP, III, 287).

Además, podríamos decir que con esta exigencia Spinoza no afirma que los hombres pierdan completamente todo su poder, sino que solo pierden el poder de defenderse, que es parte de todo el poder que poseen los hombres. En este sentido, una consecuencia que se puede inferir de estas condiciones del pacto, como también ya lo mencionamos antes, establece que los ciudadanos pueden romper el pacto si el fin para el que instituyeron el Estado, esto es, para garantizar la vida, y aumentar el bienestar y la libertad no se cumple.

En un análisis que tenga en cuenta el contexto histórico de Spinoza, y mediante una interpretación de la intencionalidad de Spinoza podríamos concluir que Spinoza, en tanto se opone al poder absoluto monárquico, también muestra su compromiso político con el partido que defiende el régimen republicano en lugar del régimen monárquico. En otras palabras, cuando Spinoza establece la democracia como la forma natural de la asociación de los individuos, que ceden su poder individual para compartir un poder común, devela su intencionalidad republicana. La pertinencia de esta inferencia reside en que, tal como lo explica Balibar, en Spinoza se evidencia una relación coherente entre su pensamiento político y sus compromisos políticos. La confirmación de esta identidad lleva a Balibar (2009) a la denominación de la política (de Spinoza), pues quiere evitar caer en distinciones entre la práctica política y la teoría política porque considera que en Spinoza no existe tal separación.

Ahora bien, volviendo a nuestro análisis de las condiciones de los individuos en el Estado, en pro de considerar por qué los ciudadanos obedecen la ley del Estado es pertinente tener presente que, de acuerdo con Spinoza, en la transición de las condiciones del estado natural a las condiciones de los ciudadanos en el Estado (TTP, XVI,193/20) no hay cambio; esta transición puede considerarse más bien en términos de continuidad, y ello a diferencia de Hobbes que, como lo explicamos antes, concibe la transición al Estado como negación de las condiciones iniciales del estado natural. Ahora bien de ésta negación tomamos dos consideraciones 
respecto a la obediencia de la ley: en primer lugar Hobbes considera que los individuos una vez que suscriben el contrato, ceden completamente su capacidad de usar la fuerza, y en adelante están obligados a obedecer la ley (Leviatán, cap. XIV y XVIII), es decir, los hombres pierden el derecho de cuestionar la ley y de plantearse la posibilidad de no obedecerla. En segundo lugar, Hobbes infiere la razón de esta situación a partir del miedo a la muerte, pues procuran su conservación en el estado de naturaleza, por lo tanto podría decirse que en Hobbes la fuerza vinculante de la ley es el miedo (Leviatán, cap. XVII).

En cambio, para Spinoza el Estado es el lugar de realización de la libertad de los individuos o del aumento de su conatus, por lo tanto no tiene sentido coartar la libertad, pero para lograr promover la libertad se requiere que los individuos actúen de acuerdo con la razón (E4P35D). Para Spinoza solo la vida en común puede garantizar el ejercicio de la libertad, es decir, "el hombre es más libre en el Estado donde obedece la ley común, que en la soledad donde solo se obedece a sí mismo" (E3P72), luego sería contradictorio presentar la obediencia de la ley como opuesta a la libertad o presentar la obediencia de la ley en términos de sometimiento acrítico.

De ahí que Spinoza piense de una forma novedosa, y para entender la novedad de su pensamiento empezamos por considerar la oposición entre obedecer la ley y ser libre. En la segunda parte de la Ética, Spinoza concluye que suele creerse, de manera equivocada, que libre es quien vive bajo su antojo, cumpliendo sus deseos $(E 3 P 2 S e)$. Por ejemplo, alguien puede obrar sin cumplir ninguna ley (un ebrio) y en consecuencia puede creer que es libre, sin darse cuenta que ese obrar puede estar determinado por sus afectos $\mathrm{y}$, por lo tanto, ese hombre no sería libre sino esclavo de sus pasiones. En ese caso, la obediencia de la ley no es la única posible causa de perdida de libertad, pues aún en el caso de aquel que solo sigue sus pulsiones, este no es libre.

Pero, Spinoza no concibe la libertad como un actuar de acuerdo con la propia voluntad, en cambio, como explicamos anteriormente, la libertad para Spinoza consiste en que el hombre obra de tal manera que es causa adecuada de sus propias acciones. Además, en la Ética, Spinoza establece otra condición para poder considerar una acción como libre. Explica que una acción también tiene que reportar utilidad (E4P20S), y que una acción es útil si aumenta el conatus, esto es, si aumenta la potencia de existir. Quizás con esta explicación se entiende mejor porqué Balibar considera el uso de la razón en Spinoza como utilitarista pero no instrumental. Esto quiere decir que las acciones libres del hombre también están determinadas por el fin, que en este caso consiste en la utilidad particular, de tal manera que un hombre es libre si es causa adecuada de sus acciones y si esas acciones son 
útiles. En caso contrario, cuando el hombre obra y sus acciones no le son útiles o no aumentan su bienestar, y al contrario con sus acciones aumenta el bienestar de otro, en ese caso se considera esclavo.

Hasta aquí podríamos decir que Spinoza piensa la libertad en el orden de los intereses particulares o desde una perspectiva ética, puesto que piensa a los hombres solo en tanto que individuos. Pero una vez hecho el pacto, se crea el orden de los intereses comunes, en ese orden instituido surge la libertad que podríamos llamar política, que en algunos casos podría entrar en contradicción con el mandato de libertad de los individuos en estado natural. No obstante, esta contradicción la resuelven los individuos en tanto que consideran que la razón para cumplir la ley, especialmente si la ley es absurda, reside en que el bien común redunda en el bien particular. Este planteamiento muestra que para Spinoza el cumplimiento de la ley no se reduce a la obediencia por miedo ni a la pérdida del derecho de pensar de los individuos. Sino que supone una comunidad donde se privilegia el ejercicio de la libre razón; en este sentido, Smith es enfático al sostener que para Spinoza "la libertad sólo se alcanza en el interior de una comunidad de personas racionales" (Smith, 2007, p.191).

La gran diferencia con Hobbes reside en que el cumplimiento de la ley es la oportunidad que tiene el hombre para ser libre, lo cual quiere decir en la lógica del pensamiento de Spinoza que sólo en el Estado el hombre podría ser causa adecuada de sus propias acciones, y sólo en el Estado podría actuar para obtener utilidad. De ahí que el cumplimiento de la ley para Spinoza supondría no un sometimiento sino una liberación. No obstante, aún podríamos preguntarnos por qué el cumplimiento de la ley hace más libres a los hombres, y la respuesta tiene que ver con dos elementos que Spinoza considera fundamentales para la libertad.

En primer lugar, la libertad supone que el individuo es causa adecuada de sus propias acciones. En segundo lugar, supone que las acciones comportan utilidad para el agente, utilidad que como lo explicamos antes tiene que ver con el aumento del conatus. Ahora bien, Spinoza piensa que en el estado natural es muy difícil que los hombres puedan realizar acciones útiles porque en el estado natural el hombre solo se obedece a sí mismo y para lograr una acción útil se requiere de otros; la situación se torna más compleja si tenemos presente que en el estado natural cada individuo emplea el derecho natural en contra de los demás. Esto significa, finalmente, que el hombre vive solo en estado natural, por eso para Spinoza en estado de naturaleza el hombre no puede ser libre porque, como lo abordamos antes, en el estado natural los hombres aún si actúan de forma racional no alcanzan la libertad. Por esta razón, para Spinoza solo en el Estado es posible la libertad y de ese modo 
se comprende mejor porque Gatens (Gatens y Lloyd,1999) sostiene que la vida en comunidad no es una opción sino una necesidad.

Otra forma de pensar este cambio es mostrar que la libertad en estado de naturaleza es abstracta, mientras que la libertad en la sociedad es concreta; es real. En consecuencia la fuerza vinculante de la norma en Spinoza, a diferencia de Hobbes (Leviatán, XIV, 31), no se reduce al miedo; también está relacionada con el deseo de libertad que tienen los hombres y ese deseo de libertad los lleva a obedecer tanto o con mayor fuerza las leyes del Estado que vivir en la soledad de acuerdo con su propio criterio.

De otra parte, la libertad implica que el hombre sea causa adecuada, lo cual significa que aunque todos los hombres tienen afectos, no todos los hombres comprenden sus afectos, por lo tanto hay una diferencia entre los afectos que los hombres experimentan sin entenderlos y los que experimentan cuando los entienden. Por lo tanto, podemos concluir con Spinoza que "solo actuamos en tanto que entendemos" (E4P24). Balibar (2009) dirá al respecto que por eso no se puede separar la razón del comportamiento, y que en esto consiste el poder de la razón.

En su optimismo político Spinoza establece una condición para la libertad: obedecer. Esto no implica que Spinoza desconozca que existen Estados y gobiernos que no tienen como fin los intereses de la sociedad, y que sólo se preocupan del bienestar del gobernante. Son Estados absolutistas donde el miedo es la única posibilidad para que sus súbditos obedezcan la ley. Si por un momento hacemos abstracción de esos Estados, y si nos ocupamos solo de los Estados que se preocupan por alcanzar "la salvación del pueblo" (TTP, XVI, 195), destacaremos que en el Estado de Spinoza existe una alternativa para obedecer la ley a la obediencia por miedo. Esto es, obedecer por libertad, lo cual parece un oxímoron; en realidad es la figura que mejor expresa el pensamiento de Spinoza, sólo el hombre que obedece es libre, porque sabe que sólo puede ser libre junto a otros hombres libres, y para vivir junto a otros hombres necesita de leyes que regulen sus relaciones. Un Estado que propicia que los ciudadanos obren libremente, lo cual implica que el soberano gobierna para el bien de todos, es un Estado libre, y sólo si cumple con este fin, a saber, que los hombres actúen libremente, es un Estado. Spinoza explica que cuando un hombre obedece a la autoridad suprema, y ésta gobierna para la salvación del pueblo, entonces esta obediencia es útil a cada hombre y a la comunidad y por esta razón deviene ciudadano libre. 


\section{La determinación del miedo}

No obstante, no todos los hombres obran conforme a su razón; las acciones de la mayoría están determinadas por sus pasiones. Luego, si nos preguntamos cómo cumplen la ley los hombres que no son causa adecuada de sus propias acciones, de los que no buscan su propia utilidad, y que, en consecuencia, no comprenden la utilidad del cumplimiento de la ley, encontramos que Spinoza no se ocupa de ellos en el TTP. Respondemos, de forma polémica, que no se ocupa de ellos porque en el TTP propone una reflexión de orden más normativo, y desde esa perspectiva solo se ocupa, según su criterio, del mejor régimen: la democracia; a lo cual, se añade, en condiciones ideales, los hombres que son causa adecuada de sus propias acciones y buscan la utilidad de sus propias acciones, esto es, que obran conforme a su razón.

Sin embargo, esto significa que Spinoza tiene en cuenta que no todos los hombres actúan de acuerdo con su razón, y aunque no lo considere en el TTP en la Ética y en el TP sí reflexiona al respecto. En la Ética, Spinoza explica ampliamente la diversidad humana, desde su comprensión de los géneros de conocimiento, y de los concomitantes grados de libertad. Explica que, en el Estado, no todos los hombres obedecen porque comprenden que obedecer les es útil; la "multitud" está determinado por sus pasiones y para que obedezca la ley es necesario someterla por el temor. De otra parte, en el $T P$, en el que se propone un análisis desde el realismo político, tal como lo explica en el prólogo, tiene en cuenta las diferencias que planteó en la Ética, y propone que para los hombres que están determinados por sus pasiones se aplica las consideraciones que tiene Hobbes sobre el gobierno para todos los hombres.

Por lo tanto, unos cumplen la ley porque comprenden su utilidad para la libertad y otros simplemente por miedo, pero el Estado tiene como uno de sus fines hacer que aun los que cumplen por miedo también cumplan la ley porque entienden su utilidad; de hecho, este es uno de los principales fines que tiene el Estado para Spinoza: lograr que todos los ciudadanos cumplan la ley por la utilidad de la ley, pues en la lógica de Spinoza esto es igual a que los hombres sean libres.

De esta manera podemos encontrar otro punto de convergencia y divergencia entre el pensamiento político de Spinoza y el pensamiento político de Hobbes. La política de Spinoza converge con la propuesta política de Hobbes respecto a la fuerza vinculante de la ley, pero sólo como una política destinada a someter a quienes no obedecen la ley porque no entienden su utilidad, y en ese sentido el miedo es el mejor instrumento para obedecer. En cambio, Spinoza se distancia de Hobbes en cuanto piensa que algunos 
ciudadanos podrían obedecer por la utilidad de la ley, en lugar de obedecer por la reducción de comportamiento al miedo. Además, Spinoza propone que si bien, en el momento instituyente es necesario establecer esta diferencia, una vez instituido el Estado, éste tiene como uno de sus fines proponer los medios para que todos los hombres sean libres en sentido político.

El hombre que se guía por su razón se da cuenta que la única posibilidad que tiene para aumentar su potencia de actuar es "allí donde los hombres tienen derechos comunes" (TP, IV, 293). Así, aunque el Estado de Spinoza tenga unas semejanzas con el Estado hobbesiano, sus visiones difieren de manera significativa. Es cierto que el sometimiento al poder por parte de la multitud, en la práctica, supone ceder gran parte de la autonomía de cada uno para poder "someterse plenamente al juicio del otro" (TP, IV, 293), lo cual parece ser contrario a la razón. Sin embargo, puesto que la razón busca aumentar el conatus, en la ponderación de los dos estados, encuentra que en el primero el hombre es mucho más susceptible de vivir sometido a las pasiones, lo cual contradice a su razón, y por lo tanto decide asociarse.

Para Spinoza es indudable que la razón aconseja la libertad y la paz, y esto sólo se obtiene en el Estado si se respetan los derechos comunes, cumpliendo las leyes. Teniendo en cuenta estas condiciones, Spinoza considera que sólo cuando el hombre se guía por la razón, y cuanto más se guíe por la razón, es más libre que en la soledad o es más libre que cuando es determinado por sus pasiones. De esta manera nadie puede llamarse libre, y actuar en contra de los demás, ni un Estado tampoco puede llamarse libre si no garantiza el bienestar común de todos los hombres.

\section{Conclusión}

A partir de lo expuesto por Spinoza en el Tratado Teológico Político en primer lugar, y posteriormente en el Tratado Político podemos concluir que el principal obstáculo que tiene que enfrentar la política es el engaño de quienes usan la política para su propio beneficio. Y ese engaño ha sido utilizado desde los romanos, que fue utilizado en le época de Spinoza, y que sigue siendo utilizado hoy.

Spinoza denuncia la estructura del engaño, pues demuestra que consiste en primer lugar en hacer creer a los hombres que la naturaleza tiene formas misteriosas de obrar que es incomprensible para la mayoría de hombres, y hoy diríamos también para las mujeres. Que solo unos pocos elegidos pueden comprender ese comportamiento misterioso, gracias a una mezcla entre inteligencia superior y el favor de los dioses, lo que da como resultado que hombres y mujeres cooptados por el miedo a lo incomprensible ceden 
no solo su poder, sino también su capacidad de juzgar, y con esto reduzcan su capacidad de existir.

Pero Spinoza sabe que cuando la multitud comprende de forma adecuada que no hay tal poder mayor o inteligencia superior de los políticos y gobernantes de turno, sino que el aumento de poder y la agudeza de inteligencia responden a la fuerza deliberativa que proviene del encuentro de todos los individuos en la sociedad, entonces empezará la verdadera democracia, puesto que ella emerge de la libertad.

\section{Referencias bibliográficas}

Balibar, E. (2011). Spinoza y la Política. Buenos Aires, Argentina: Prometeo.

Balibar, E. (2009). Spinoza: de la individualidad a la transindividualidad. Córdoba, Argentina: Brujas.

Gatens, M. y Lloyd, G. (1999). Collective Imaginings. Spinoza past and present. Londres, Inglaterra: Routledge.

Hobbes, T. [Leviatán] (2007). Leviatán. Madrid, España: Losada.

Peña, V. (1974). El materialismo de Spinoza: Ensayo sobre la ontología spinozista.

Madrid, España: Ediciones de la Revista de Occidente.

Smith, S. (2007). Spinoza y el libro de la vida. Madrid, España: Biblioteca Nueva. Spinoza, B. [E] (1999). Ética. Madrid, España: Alianza.

Spinoza, B. [TTP] (1996). Tratado Teológico Político y Tratado Político. (E. Tierno Galván, Trad.). Madrid, España: Tecnos.

Spinoza, B. [TP] (2010). Tratado Político. (A. Domínguez, Trad.). Madrid, España: Alianza Editorial. 